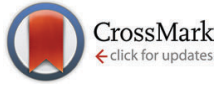

Cite this: Phys. Chem. Chem. Phys., $2016,18,26998$

DOI: $10.1039 / \mathrm{c} 6 \mathrm{cp} 90232 d$

www.rsc.org/pccp

\section{Correction: Effect of lipid head group interactions on membrane properties and membrane-induced cationic $\beta$-hairpin folding}

\author{
Sai J. Ganesan, ${ }^{a}$ Hongcheng $\mathrm{Xu}^{\mathrm{b}}$ and Silvina Matysiak*ab
}

Correction for 'Effect of lipid head group interactions on membrane properties and membrane-induced cationic $\beta$-hairpin folding' by Sai J. Ganesan et al., Phys. Chem. Chem. Phys., 2016, 18, 17836-17850.

The authors would like to correct an error in the abstract that was introduced during production of the article. The first sentence of the abstract in the published article should be replaced with that shown in the correct version below:

Membrane interfaces (mIFs) are ubiquitous components of living cells and are host to many essential biological processes. One key characteristic of mIFs is the dielectric gradient and, subsequently, electrostatic potential that arises from dipolar interactions in the head group region. In this work, we present a coarse-grained (CG) model for anionic and zwitterionic lipids that accounts for dipolar intricacies in the head group region. Prior work on adding dipolar interactions in a coarse grained (CG) model for peptides enabled us to achieve a/b secondary structure content de novo, without any added bias. We have now extended this idea to lipids. To mimic dipolar interactions, two dummy particles with opposite charges are added to CG polar beads. These two dummy charges represent a fluctuating dipole that introduces structural polarization into the head group region. We have used POPC (1-palmitoyl-2-oleoyl-sn-glycero-3-phosphocholine) and POPS (1-palmitoyl-2-oleoyl-sn-glycero-3-phospho-L-serine) as our model lipids. We characterize structural, dynamic, and dielectric properties of our CG bilayer, along with the effect of monovalent ions. We observe head group dipoles to play a significant role in membrane dielectric gradient and lipid clustering induced by dipoledipole interactions in POPS lipids. In addition, we have studied membrane-induced peptide folding of a cationic antimicrobial peptide with anticancer activity, SVS-1. We find that membrane-induced peptide folding is driven by both (a) cooperativity in peptide self-interaction and (b) cooperativity in membrane-peptide interaction. In particular, dipolar interactions between the peptide backbone and lipid head groups contribute to stabilizing folded conformations.

The Royal Society of Chemistry apologises for these errors and any consequent inconvenience to authors and readers.

\footnotetext{
${ }^{a}$ Fischell Department of Bioengineering, University of Maryland, College Park, Maryland, USA. E-mail: matysiak@umd.edu; Tel: +1 3014050313

${ }^{b}$ Biophysics Program, University of Maryland, College Park, Maryland, USA
} 